\title{
LO QUE EL VIENTO NO SE LLEVÓ. ANÁLISIS DE SITIOS DE SUPERFICIE EN LA ESTEPA FUEGUINA.
}

\author{
FERNANDO SANTIAGO Y Y JIMENA ORÍA"
}

\begin{abstract}
RESUMEN
Se presenta información de dos sitios arqueológicos de superficie del norte de Tierra del Fuego que se encuentran en puntos altos del paisaje expuestos a la acción eólica. Este trabajo informa los resultados obtenidos en el análisis tecnológico de los materiales líticos de ambos sitios así como también información arqueofaunística. Estos nuevos datos constituyen un aporte al conocimiento de las poblaciones que habitaron la zona durante el Holoceno tardío.
\end{abstract}

PALABRAS CLAVES: Sitios de superficie, Tierra del Fuego, procesos postdepositacionales.

UNBLOWN BY THE WIND. ANALYSIS OF SURFICIAL SITES IN THE FUEGIAN STEPPE.

\section{SUMMARY}

This paper present information of two surficial archaeological sites of the north of the Tierra del Fuego, which are located in high points of the landscape, exposed to the aeolian action. The aim of the current paper is to show the results obtained in the technological analysis of the lithic materials of both sites as well as the archaeofaunal information. These new data represent a contribution to the knowledge of the populations that inhabited the zone during the Late Holocene.

KEYWORDS: Surficial sites, Tierra del Fuego, posdepositional processes.

\section{INTRODUCCIÓN}

Los depósitos o sitios de superficie (Holdaway et al. 2004) también llamados sitios someros (Zárate et al. 2000-2002) son aquellos en los que el material arqueológico está totalmente expuesto a las condiciones subaéreas, ya sea por erosión de la matriz, por sedimentación escasa o por diversas disturbaciones. Los mismos carecen de una estratigrafía en el sentido convencional de Butzer (1982:104), por lo tanto es difícil asignarles una cronología.

Los sitios de superficie representan a menudo períodos largos de depositación, es por ello que la proximidad espacial de ítems arqueológicos no es

* Becario CONICET Lab. de Geología del Cuaternario. CADIC. B. Houssay 200. Ushuaia. fsantiago@cadic.gov.ar

* Lab. de Geología del Cuaternario. CADIC. B. Houssay 200. Ushuaia. jimenaoria@cadic.gov.ar 
ninguna garantía de sincronía. Centenares de años pueden separar características espaciales apartadas por escasos centímetros, y no en todos los casos los artefactos pueden fecharse directamente. Sin embargo existen algunas técnicas para diferenciar conjuntos de artefactos referentes a acontecimientos u ocupaciones específicas, el reensamblaje es un ejemplo (Ramos y Merenzon 2004). En la mayor parte de los casos se debe asumir que un sitio de superficie puede derivar de más de un evento de ocupación, y a menudo muchos eventos separados u ocupaciones.

La pérdida de información en yacimientos de superficie en el norte de Tierra del Fuego es alta, debido a la fuerte dinámica de los vientos predomi- nantes (Massone et al. 1993; Prieto 1997; Favier Dubois y Borrero 2005), pero consideramos que no por ello debe menospreciarse la información contenida en estos depósitos.

En este trabajo se presenta un análisis preliminar de los materiales líticos y óseos hallados en los sitios Avilés 1 y Herradura 1(Fig. 1), lo realizado se enmarca en el proyecto "La ocupación humana en el norte de Tierra del Fuego durante el Holoceno medio y tardío. Su vinculación con el paisaje". Entre los objetivos del proyecto marco se encuentra analizar el tipo de asentamientos en cuanto a su localización y la forma de utilización del espacio por parte de grupos humanos en el pasado.

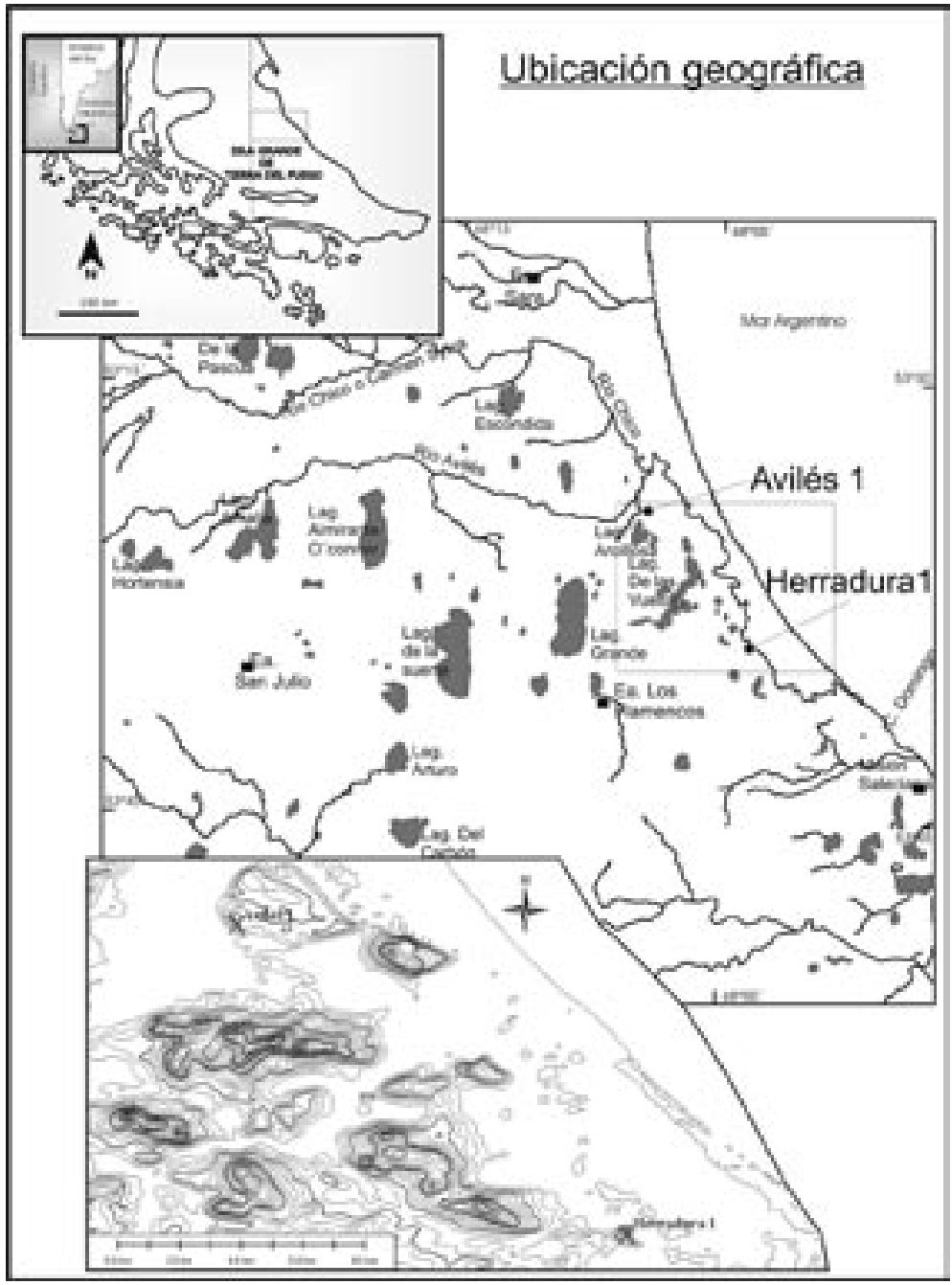

Fig. 1. Mapa de ubicación geográfica de los sitios en la zona de estudio. 


\section{METODOLOGÍA}

Los sitios han sido localizados a partir de prospecciones sistemáticas en la cuenca del Río Chico (Santiago et al. 2006); los mismos fueron geoposicionados utilizando GPS's diferenciales (Ashtech 2, Mobil Mapper), lo cual permite tener un error horizontal menor a diez centímetros y vertical menor a un metro.

Todos los ítems arqueológicos se han levantado con el sistema de Estación Total (Pentax $\mathrm{R}$-326). Se siguió esta metodología porque facilita reconstruir con mayor detalle la topografía de los sitios y obtener la ubicación exacta de todos los materiales arqueológicos prescindiendo de un cuadriculado.

El análisis tecno-tipológico de los materiales líticos se realizó siguiendo a Aschero (1975) y Orquera y Piana (1986). Las variables analizadas fueron: para núcleos, designación morfológica y tipos de materias primas; para desechos de talla, características litométricas y litotécnicas, materias primas y estado de fragmentación; y para artefactos formatizados, características litométricas, litotécnicas y tipos de materias primas.

Los restos óseos hallados fueron analizados siguiendo los lineamientos metodológicos generales ya convencionales (Grayson 1984; Mengoni Goñalons 1999; Lyman 1994) buscando determinar para cada espécimen además de la asignación taxonómica y anatómica, lateralidad, grado de meteorización, presencia de marcas o huellas de algún tipo, tipo de fractura presente $y$, en cuanto fuera posible, establecer la edad de muerte.

\section{CARACTERÍSTICAS AMBIENTALES}

Los sitios analizados se encuentran en la cuenca del río Chico, en la región septentrional de la Isla Grande de Tierra del Fuego, entre el cabo San Sebastián y el cabo Domingo. El paisaje corresponde a la estepa fueguina. Esta unidad de vegetación que ocupa el norte de Tierra del Fuego es equivalente a la estepa magallánica húmeda en su porción continental. El aspecto que presenta es homogéneo, predominando un paisaje abierto de extensas llanuras y mesetas recortadas, con una cubierta vegetal de especies herbáceas y arbustivas (Oliva et al. 2001).
El viento fuerte y constante constituye una singularidad de la estepa, de hecho se podría decir que la estepa es viento. Porque ha sido el gran creador del paisaje, después de las glaciaciones y hasta el presente. La gran mayoría de los sedimentos, terciarios o cuaternarios, fueron cubiertos por una capa de un sedimento fino y limoso que a modo de manto transportado por el viento, cubrió el paisaje y sirvió de sustrato primario a los suelos que sustentan la vegetación de la estepa (Bianciotto 2006).

Fisonómicamente es una estepa graminosa de coirón fueguino (Festuca gracillima) con áreas dominadas por mata negra fueguina (Chilliotrichum diffusum) y otras en las cuales existen arbustos rojizos, rastreros de murtilla (Empetrum rubrum). Existen también praderas de pastos cortos que se alternan con los coironales en forma de mosaico (Oliva et al. 2001).

La estepa evolucionó con baja presión de pastoreo asociados a dos herbívoros nativos: Ctenomys magellanicus y Lama guanicoe. La historia de pastoreo intenso se inició a fines de 1800 con la introducción de las ovejas por inmigrantes. La vegetación de la estepa fue modificada por la ganadería. En algunas áreas el exceso de pastoreo y pisoteo de los ovinos resulta en una disminución de los arbustos, los cuales están siendo reemplazados por pastizales de pastos cortos. En contraposición, en otras partes, el pastoreo esporádico conduce a la formación de manchones densos de arbustos (Bianciotto 2006).

\section{UBICACIÓN Y DESCRIPCIÓN GENERAL DE LOS SITIOS}

El sitio Avilés 1 (53 34' 22.82514"S; $68^{\circ}$ 04' 41.07712"O) (Fig. 1) se encuentra ubicado sobre la cima de una colina de sedimentitas del Terciario, orientado hacia la planicie de inundación del río homónimo y dista unos 3000 metros en línea recta de la costa actual. Se encuentra a 41,5 msnm y la superficie del sitio es de $1462 \mathrm{~m}^{2}$. Los materiales arqueológicos del sitio Avilés 1 (A1 en adelante) se encontraron apoyados directamente sobre sedimentos del Terciario, pero en algunos sectores del sitio se observaban remanentes o testigos de los sedimentos eólicos que los contenían antes de su exposición. 
Tabla 1. Distribución de clases de instrumentos y materias primas en A1.

\begin{tabular}{|c|c|c|c|c|c|c|c|c|c|c|c|}
\hline \multirow{2}{*}{\multicolumn{2}{|c|}{ Tipos }} & \multirow{2}{*}{$\mathbf{N}$} & \multicolumn{7}{|c|}{ Materias Primas* } & \multirow{2}{*}{ Total } & \multirow{2}{*}{$\%$} \\
\hline & & & $\mathrm{Ri}$ & $\mathrm{Cal}$ & Bas & Hor & $\mathrm{Ar}$ & $\mathrm{Cu}$ & IND & & \\
\hline \multirow{5}{*}{ 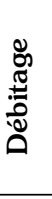 } & Nódulo & & 4 & 1 & 1 & 0 & 0 & 0 & 2 & 8 & 4,8 \\
\hline & Núcleo & & 14 & 9 & 5 & 0 & 0 & 1 & 0 & 29 & 17,4 \\
\hline & Desecho indiferenciado & 146 & 6 & 4 & 3 & 0 & 0 & 0 & 0 & 13 & 7,8 \\
\hline & Lasca & & 52 & 26 & 12 & 0 & 0 & 0 & 2 & 92 & 55,1 \\
\hline & Lámina & & 3 & 1 & 0 & 0 & 0 & 0 & 0 & 4 & 2,4 \\
\hline \multirow{16}{*}{ 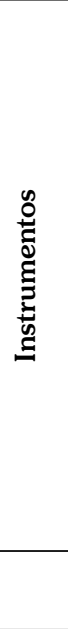 } & Alisador & \multirow{14}{*}{21} & 0 & 0 & 0 & 0 & 1 & 0 & 0 & 1 & 0,6 \\
\hline & Biface & & 1 & 1 & 0 & 0 & 0 & 0 & 0 & 2 & 1,2 \\
\hline & Biface incompleto & & 1 & 0 & 0 & 0 & 0 & 0 & 0 & 1 & 0,6 \\
\hline & Bola & & 0 & 0 & 0 & 2 & 0 & 0 & 0 & 2 & 1,2 \\
\hline & Cepillo & & 1 & 0 & 0 & 0 & 0 & 0 & 0 & 1 & 0,6 \\
\hline & Percutor & & 2 & 0 & 0 & 0 & 0 & 0 & 1 & 3 & 1,8 \\
\hline & Poliedro & & 1 & 0 & 0 & 0 & 0 & 0 & 0 & 1 & 0,6 \\
\hline & Raedera borde bifacetado & & 0 & 1 & 0 & 0 & 0 & 0 & 0 & 1 & 0,6 \\
\hline & Raedera convergente & & 1 & 0 & 0 & 0 & 0 & 0 & 1 & 2 & 1,2 \\
\hline & Raedera convergente alterna & & 0 & 0 & 1 & 0 & 0 & 0 & 0 & 1 & 0,6 \\
\hline & Raedera doble & & 1 & 0 & 0 & 0 & 0 & 0 & 0 & 1 & 0,6 \\
\hline & Raedera simple recta & & 0 & 1 & 0 & 0 & 0 & 0 & 0 & 1 & 0,6 \\
\hline & Guijarro c/retoque apical & & 0 & 1 & 1 & 0 & 0 & 0 & 0 & 2 & 1,2 \\
\hline & Lasca c/retoque & & 0 & 1 & 1 & 0 & 0 & 0 & 0 & 2 & 1,2 \\
\hline & $\begin{array}{r}\text { TOTAL } \\
\end{array}$ & 167 & 87 & 46 & 24 & 2 & 1 & 1 & 6 & 167 & 100 \\
\hline & $\%$ & 100 & 52,1 & 27,5 & 14,4 & 1,2 & 0,6 & 0,6 & 3,6 & 100 & \\
\hline
\end{tabular}

${ }^{*} \mathrm{Ri}=$ Riolita, $\mathrm{Cal}=$ Calcedonia, $\mathrm{Bas}=$ Basalto, Hor $=$ Hornblendita, $\mathrm{Ar}=$ Arenisca $\mathrm{Cu}=\mathrm{Cuarzo}$, IND= Indeterminadas

El sitio Herradura $1\left(53^{\circ} 39^{\prime} 48.38^{\prime \prime} \mathrm{S}\right.$ $67^{\circ} 57^{\prime} 48.83$ "O) (Fig. 1) también se encuentra ubicado en la cima de otra colina de origen Terciario a 12 $\mathrm{km}$ al sureste de A1, y tiene una amplia visibilidad hacia el este sobre la planicie de inundación del río Chico y del océano Atlántico, el cual se encuentra a $2800 \mathrm{~m}$ en línea recta. El sitio de halla a 52,5 msnm y tiene una superficie de $1799 \mathrm{~m}^{2}$.

De la misma manera que en A1 los materiales arqueológicos de Herradura 1 (H1 en adelante) se encontraban apoyados directamente sobre la roca de origen Terciario. En este caso se pudo observar que en el lapso de 7 meses entre la primera y la segunda visita al sitio, el viento provocó una deflación de unos $980 \mathrm{~m}^{2}$ (diferencia medida con GPS's diferenciales). En la primer visita el sitio se clasificó como una concentración, con menos de 20 ítems líticos (sensu Borrero et al. 1992) en superficie; luego, en la segunda visita se registraron 464 ítems arqueológicos. Esta intensa dinámica erosiva ya había sido observada en otros sectores de la isla (Lanata 1993; Massone et al. 1993; Favier Dubois y Borrero 2005).

\section{SITIO AVILÉS 1}

Análisis lítico

En el sitio A1 fue recuperada una muestra de 167 especímenes líticos. En la Tabla 1 se puede observar la distribución de las frecuencias de las categorías artefactuales teniendo en cuenta las materias primas empleadas tanto para el débitage como para los instrumentos.

Tabla 2: Tipos de lascas en A1.

\begin{tabular}{|c|c|c|}
\hline Tipo de lasca & $\mathrm{N}$ & $\%$ \\
\hline Arista recta & 1 & 1 \\
\hline Dorso preparado & 2 & 2 \\
\hline Lámina de arista & 2 & 2 \\
\hline Lámina secundaria & 2 & 2 \\
\hline Angular inclinada & 3 & 3 \\
\hline Dorso natural & 10 & 10 \\
\hline Angular oblicua & 11 & 11 \\
\hline Primaria & 15 & 16 \\
\hline Secundaria & 19 & 20 \\
\hline Angular recta & 31 & 32 \\
\hline TOTAL & 96 & 100 \\
\hline
\end{tabular}




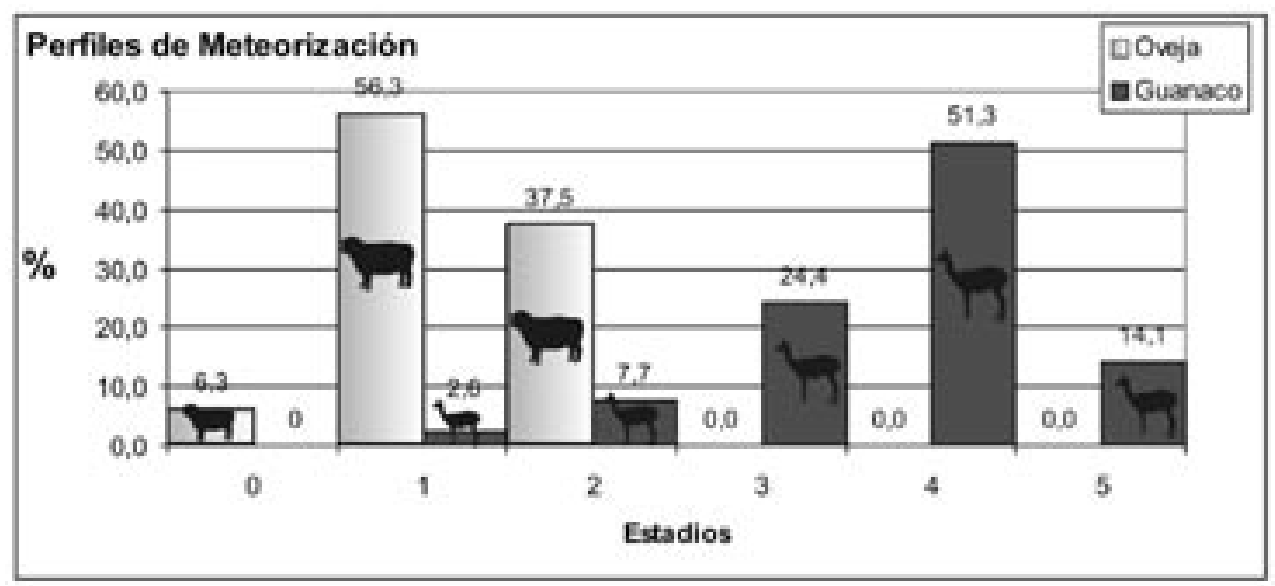

Fig. 2. Perfiles de meteorización de guanacos y ovejas en A1.

El 51\% del total de las lascas conservan corteza. $\mathrm{Al}$ discriminar por materias primas, la corteza se observa en el 63,64\% de las lascas de Riolita y en el $50 \%$ de las lascas de calcedonia y basalto.

Las frecuencias de los distintos tipos de lascas puede observarse en la Tabla 2.

Otro aspecto que se consideró para las lascas fue el tipo de talón. El 22\% de las lascas no presenta talón, de las restantes (enteras y fragmentadas con talón, $\mathrm{N}=75$ ), el $44 \%$ es de tipo liso, el talón preparado representa el 15\% y el liso natural el 13\%, el diedro $11 \%$, el de tipo filiforme $9 \%$, y por último, los menos representados fueron el talón de tipo natural y el de tipo facetado con un $5 \%$ cada uno.

En la categoría núcleos ( $\mathrm{N}=29)$, la forma base exclusiva es la de los rodados (100\%). El 45\% de los núcleos son de talla bipolar, el 38\% son amorfos, el $10 \%$ son del tipo globuloso y el $7 \%$ discoidales. La totalidad de los núcleos se encuentran enteros.

Análisis faunístico

En el sitio A1 el NISP total es 104, valor que se agrupa en tres taxa: Lama guanicoe (NISP = $78)$, Ovis aries (NISP $=16$ ) y ave (NISP $=6$ ). Hay 4 especímenes que permanecen indeterminados. Para el guanaco se determinó un número mínimo de individuos (MNI) de 6. Para oveja el MNI es de 1, como así también en las aves (Tabla 3).

En cuanto a la meteorización, en base a los estadíos de Behrensmeyer (1978), se logró desdoblar la muestra en dos perfiles, uno para guanaco y otro para oveja (Fig. 2).
En la Tabla 4 se pueden observar las frecuencias de las marcas tafonómicas identificadas en huesos de guanaco y oveja.

Las marcas de origen antrópico se encuentran en 3 especímenes de guanaco. Son marcas de corte (2) una sobre la sección lateral proximal de un

Tabla 3. Frecuencia de partes esqueletarias en A1.

\begin{tabular}{|c|c|c|c|c|c|c|c|c|c|}
\hline & \multicolumn{5}{|c|}{ GUANACO } & \multicolumn{2}{|c|}{ OVEJA } & \multicolumn{2}{|c|}{ AVE } \\
\hline Espécimen & NISP & MNI & MNE & MAU & $\% \mathrm{MAU}$ & NISP & MNI & NISP & MNI \\
\hline $2^{\circ}$ Molar & 2 & 1 & - & - & - & 0 & 0 & 0 & 0 \\
\hline $3^{\circ}$ Molar & 2 & 1 & - & - & - & 0 & 0 & 0 & 0 \\
\hline Mandíbula & 1 & 1 & 1 & 0,5 & 14,3 & 2 & 1 & 0 & 0 \\
\hline Vrt. indet. & 2 & 1 & 2 & 0,1 & 2,1 & 0 & 0 & 0 & 0 \\
\hline Vrt. toráxica & 1 & 1 & 1 & 0,1 & 2,4 & 0 & 0 & 0 & 0 \\
\hline Costilla & 1 & 1 & 1 & 0 & 1,2 & 7 & 1 & 1 & 1 \\
\hline Escápula & 3 & 2 & 3 & 1,5 & 42,9 & 0 & 0 & 0 & 0 \\
\hline Húmero & 9 & 6 & 7 & 3,5 & 100.0 & 1 & 1 & 1 & 1 \\
\hline Radiocúbito & 8 & 5 & 7 & 3,5 & 100 & 0 & 0 & 0 & 0 \\
\hline Metacarpo & 4 & 3 & 4 & 2 & 57,1 & 0 & 0 & 0 & 0 \\
\hline Pelvis & 1 & 1 & 1 & 0,5 & 14,3 & 0 & 0 & 0 & 0 \\
\hline Fémur & 6 & 3 & 5 & 2,5 & 71,4 & 1 & 1 & 1 & 1 \\
\hline Tibia & 8 & 6 & 7 & 3,5 & 100 & 1 & 1 & 0 & 0 \\
\hline Metatarso & 4 & 2 & 4 & 2 & 57,1 & 1 & 1 & 0 & 0 \\
\hline Carpiano & 3 & 2 & 3 & 0,2 & 6,1 & 0 & 0 & 0 & 0 \\
\hline Astrágalo & 2 & 1 & 2 & 1 & 28,6 & 0 & 0 & 0 & 0 \\
\hline Metapodio & 5 & 3 & 5 & 2,5 & 71,4 & 0 & 0 & 0 & 0 \\
\hline Falange $1^{\circ}$ & 1 & 1 & 1 & 0,1 & 3,6 & 0 & 0 & 0 & 0 \\
\hline Frag. diaf. & 15 & - & - & - & - & 0 & 0 & 0 & 0 \\
\hline Esternebra & 0 & 0 & 0 & 0 & 0 & 1 & 1 & 0 & 0 \\
\hline Toráxcica & 0 & 0 & 0 & 0 & 0 & 2 & 1 & 0 & 0 \\
\hline Coracoides & - & - & - & - & - & 0 & 0 & 1 & 1 \\
\hline Cúbito & - & - & - & - & - & 0 & 0 & 2 & 1 \\
\hline TOTAL & 78 & 6 & - & - & - & 16 & 1 & 6 & 1 \\
\hline
\end{tabular}


Tabla 4. Marcas tafonómicas en A1.

\begin{tabular}{|l|l|c|c|}
\hline \multicolumn{1}{|c|}{ Avilés 1 } & $\mathrm{N}$ & $\%$ \\
\cline { 2 - 4 } & Raíces & 53 & 68 \\
\cline { 2 - 4 } & Roedor & 25 & 32 \\
\cline { 2 - 4 } & Carnívoros & 7 & 9 \\
\cline { 2 - 4 } & Pisoteo & 0 & 0 \\
\cline { 2 - 4 } & TOTAL & 78 & 100 \\
\hline \multirow{2}{*}{} & Raíces & 2 & 12,5 \\
\cline { 2 - 4 } & Roedor & 1 & 6,25 \\
\cline { 2 - 4 } & Carnívoros & 0 & 0 \\
\cline { 2 - 4 } & Pisoteo & 0 & 0 \\
\cline { 2 - 4 } & TOTAL & 16 & 100 \\
\hline
\end{tabular}

radiocúbito y otra sobre la cara anterior y proximal de una falange $1^{\text {era }}$. También se detectó un negativo de lascado (1) sobre la porción distal de la diáfisis de un metacarpo. No se observaron huesos quemados o calcinados.

En lo referente a la presencia de fracturas en la muestra de guanaco, de los 78 especímenes, 30 están enteros, y los restantes 48 se encuentran con diferentes grados de fragmentación observables en la Figura 3.

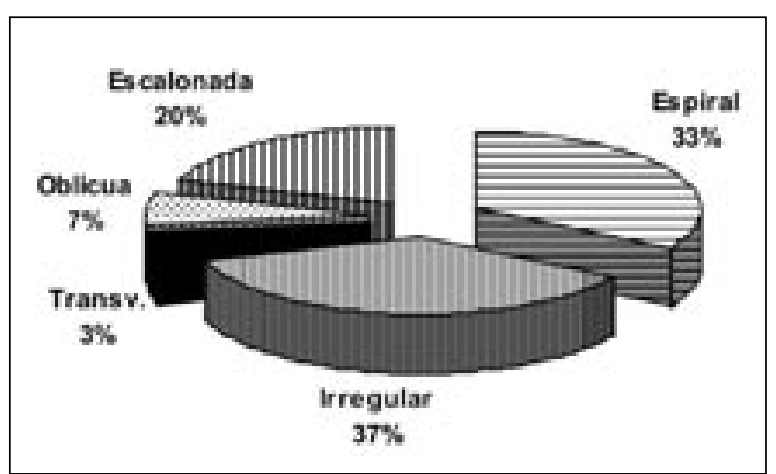

Fig. 3. Tipos de fractura en huesos de guanaco del sitio A1.

\section{SITIO HERRADURA 1}

\section{Análisis lítico}

La muestra recolectada y analizada en H1 es de 446 artefactos líticos. En la Tabla 5 se observan los detalles de las categorías artefactuales teniendo en cuenta las materias primas empleadas tanto para el débitage como para los instrumentos.

Tabla 5. Distribución de clases de instrumentos y materias primas en H1.

\begin{tabular}{|c|c|c|c|c|c|c|c|c|c|c|c|}
\hline \multirow{2}{*}{\multicolumn{2}{|c|}{ Tipos }} & \multirow{2}{*}{$\mathrm{N}$} & \multicolumn{7}{|c|}{ Materias Primas" } & \multirow{2}{*}{ Total } & \multirow{2}{*}{$\%$} \\
\hline & & & $\mathbf{R i}$ & Cal & Bas & Mgr & $\mathrm{Ar}$ & $\mathrm{Cu}$ & IND & & \\
\hline \multirow{6}{*}{ 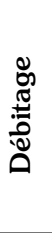 } & Nódulo & \multirow{6}{*}{421} & 2 & 2 & 2 & 2 & 2 & 1 & 3 & 14 & 3,1 \\
\hline & Núcleo & & 31 & 12 & 9 & 5 & 0 & 31 & 9 & 97 & 21,7 \\
\hline & Desecho indiferenciado & & 21 & 25 & 16 & 2 & 0 & 2 & 4 & 70 & 15,7 \\
\hline & Lasca & & 110 & 69 & 28 & 0 & 0 & 7 & 3 & 217 & 48,7 \\
\hline & Lámina & & 2 & 3 & 1 & 0 & 0 & 0 & 0 & 6 & 1,3 \\
\hline & Microlasca & & 6 & 7 & 4 & 0 & 0 & 0 & 0 & 17 & 3,8 \\
\hline \multirow{18}{*}{ 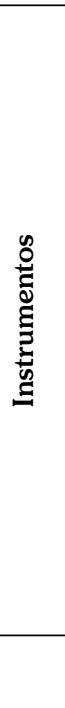 } & Biface incompleto & \multirow{16}{*}{25} & 1 & 0 & 0 & 0 & 0 & 0 & 0 & 1 & 0,2 \\
\hline & Cepillo & & 1 & 0 & 0 & 0 & 0 & 0 & 0 & 1 & 0,2 \\
\hline & Percutor & & 1 & 0 & 0 & 0 & 0 & 1 & 0 & 2 & 0,4 \\
\hline & Raedera alternante & & 1 & 0 & 0 & 0 & 0 & 0 & 0 & 1 & 0,2 \\
\hline & Raedera foliácea doble & & 0 & 0 & 1 & 0 & 0 & 0 & 0 & 1 & 0,2 \\
\hline & Raedera convergente & & 2 & 0 & 0 & 0 & 0 & 0 & 1 & 3 & 0,7 \\
\hline & Raedera simple & & 0 & 0 & 1 & 0 & 0 & 0 & 0 & 1 & 0,2 \\
\hline & Raedera doble & & 1 & 0 & 1 & 0 & 0 & 0 & 0 & 2 & 0,4 \\
\hline & Raedera simple convexa & & 1 & 1 & 1 & 0 & 0 & 0 & 0 & 3 & 0,7 \\
\hline & Raedera simple recta & & 0 & 0 & 1 & 0 & 0 & 0 & 0 & 1 & 0,2 \\
\hline & Lasca c/retoque & & 1 & 3 & 0 & 0 & 0 & 0 & 0 & 4 & 0,9 \\
\hline & Bec distal & & 1 & 0 & 0 & 0 & 0 & 0 & 0 & 1 & 0,2 \\
\hline & Denticulado simple & & 1 & 0 & 0 & 0 & 0 & 0 & 0 & 1 & 0,2 \\
\hline & Punta triangular & & 1 & 0 & 0 & 0 & 0 & 0 & 0 & 1 & 0,2 \\
\hline & Raspador circular & & 0 & 0 & 1 & 0 & 0 & 0 & 0 & 1 & 0,2 \\
\hline & Raspador unguiforme & & 0 & 0 & 1 & 0 & 0 & 0 & 0 & 1 & 0,2 \\
\hline & TOTAL & 446 & 184 & 122 & 67 & 9 & 2 & 42 & 20 & 446 & 100 \\
\hline & $\%$ & 100 & 41,3 & 27,4 & 15,0 & 2,0 & 0,4 & 9,4 & 4,5 & 100 & \\
\hline
\end{tabular}

${ }^{*} \mathrm{Ri}=$ Riolita, $\mathrm{Cal}=$ Calcedonia, Bas $=$ Basalto, Mgr $=$ Microgranito, Ar= Arenisca, $\mathrm{Cu}=$ Cuarzo, $\mathrm{IND}=$ Indeterminadas. 
Tabla 6. Tipos de lascas en H1.

\begin{tabular}{|l|cc|}
\hline \multicolumn{1}{|c|}{ Tipo de lasca } & $\mathrm{N}$ & $\%$ \\
\hline Lámina secundaria & 1 & 0,4 \\
Lámina de arista & 2 & 0,9 \\
Dorso preparado & 3 & 1,3 \\
Lámina primaria & 3 & 1,3 \\
Punta de arista recta & 5 & 2,2 \\
Arista recta & 6 & 2,7 \\
Angular inclinada & 8 & 3,6 \\
Angular oblicua & 11 & 4,9 \\
Dorso natural & 16 & 7,2 \\
Secundaria & 72 & 8,5 \\
Primaria & 77 & 32,3 \\
Angular recta & 223 & 34,5 \\
\hline \multicolumn{2}{|c|}{ TOTAL } & \\
\hline
\end{tabular}

$\mathrm{Al}$ igual que en $\mathrm{A} 1$, en el sitio $\mathrm{H} 1$ se calculó el índice de corteza de las lascas. Sobre el total de las lascas el 53,37\% tiene presencia de corteza. Al separar por materias primas, el 66,67\% de las lascas de cuarzo tienen remanentes de corteza, le siguen las lascas de riolita y de basalto con el $50 \%$ y por último la calcedonia con el 46,81\%.

Las frecuencias de los distintos tipos de lascas puede observarse en la Tabla 6.

Dentro de la categoría de desechos conformada por la suma de lascas, microlascas y láminas ( $\mathrm{N}=240)$, el 34\% no tiene talón. Del 66\% restante $(\mathrm{N}=159)$, el tipo de talón predominante es el liso, representado por el $28 \%$; le sigue el tipo natural con el $21 \%$, el liso natural con $19 \%$, los tipos filiforme y diedro con el 11\% cada uno, por último los tipos facetado y preparado están representados por el $4 \%$ cada uno.

Tabla 7: Frecuencia de partes esqueletarias en $\mathrm{H} 1$.

\begin{tabular}{|l|ccccc|cc|}
\hline \multicolumn{7}{|c|}{ GUANACO } & \multicolumn{2}{c|}{ CETACEO } \\
\hline Especimen & NISP & MNI & MNE & MAU & $\%$ MAU & NISP & MNI \\
\hline Astrágalo & 1 & 1 & 1 & 0,50 & 33,3 & 0 & 0 \\
Calcáneo & 1 & 1 & 1 & 0,50 & 33,3 & 0 & 0 \\
Costilla & 1 & 1 & 1 & 0,04 & 2,8 & 1 & 1 \\
Esternebras & 1 & 1 & 1 & 0,17 & 11,1 & 0 & 0 \\
Falange 1 & 1 & 1 & 1 & 0,13 & 8,3 & 0 & 0 \\
Fémur & 3 & 2 & 3 & 1,50 & 100,0 & 0 & 0 \\
Húmero & 2 & 2 & 2 & 1,00 & 66,7 & 0 & 0 \\
Metacarpo & 2 & 2 & 2 & 1,00 & 66,7 & 0 & 0 \\
Radiocúbito & 1 & 1 & 1 & 0,50 & 33,3 & 0 & 0 \\
Tibia & 3 & 2 & 3 & 1,50 & 100,0 & 0 & 0 \\
Mandíbula & 1 & 1 & 1 & 0,5 & 33,3 & 0 & 0 \\
\hline \multicolumn{1}{|c|}{ TOTAL } & 17 & 2 & 17 & -- & -- & 1 & 1 \\
\hline
\end{tabular}

En lo referente a los núcleos, la forma base exclusiva es la de los rodados (100\%). El 82\% de los núcleos son de talla bipolar, el 10\% son amorfos, el $6 \%$ son del tipo globuloso y por último el 1\% discoidales. La totalidad de los núcleos se encuentran enteros. La mayor parte de los núcleos solamente fueron testeados y descartados por su mala calidad o por la presencia de imperfecciones.

Análisis faunístico

El NISP total de este sitio es bajo, tan sólo 18 especimenes de los cuales el 94\% corresponde a L. guanicoe $(\mathrm{N}=17)$, con un $\mathrm{MNI}$ de 2 guanacos (Tabla 7), el restante 6\% corresponde a una costilla de cetáceo. Sobre la misma se observa en su parte proximal una zona en la cual ha sido biselada.

Del resto de la muestra, en su totalidad guanaco, el alto grado de meteorización impidió que los especimenes fueran levantados del terreno a excepción de dos casos: un astrágalo y una epífisis distal de tibia con posibles marcas de pulido. Todos los especimenes fueron determinados y realizadas todas las observaciones en el campo, ya que al intentar manipularlos estos se pulverizaban. La muestra se asigno en su totalidad a individuos adultos.

El perfil de meteorización realizado determinó que el $82 \%$ de la muestra presenta un estadío 5 y los restantes especímenes se reparten en los estadíos 3 y 4 . Las improntas de raíces estaban presentes en el $100 \%$ de la muestra y no se observaron marcas producidas por carnívoros o por roedores.

\section{DISCUSIÓN}

El estudio de los sitios muestra que la erosión eólica actual ha sido un agente de gran importancia en la alteración de los mismos. Los vientos predominantes provienen del oeste (con velocidades que a veces superan los $100 \mathrm{~km} / \mathrm{h}$ ) acrecentando las cárcavas hacia el este. Este agente altera y selecciona el tamaño de los artefactos, en algunos casos desplazándolos de su posición original. Este proceso ya se ha descrito en Massone et al. (1993) y Prieto (1997).

Trabajos experimentales desarrollados en la estepa fueguina por Massone et al. (1993) en los cuales se replicaron procesos culturales, demostraron que hay una dispersión de los materiales arqueoló- 


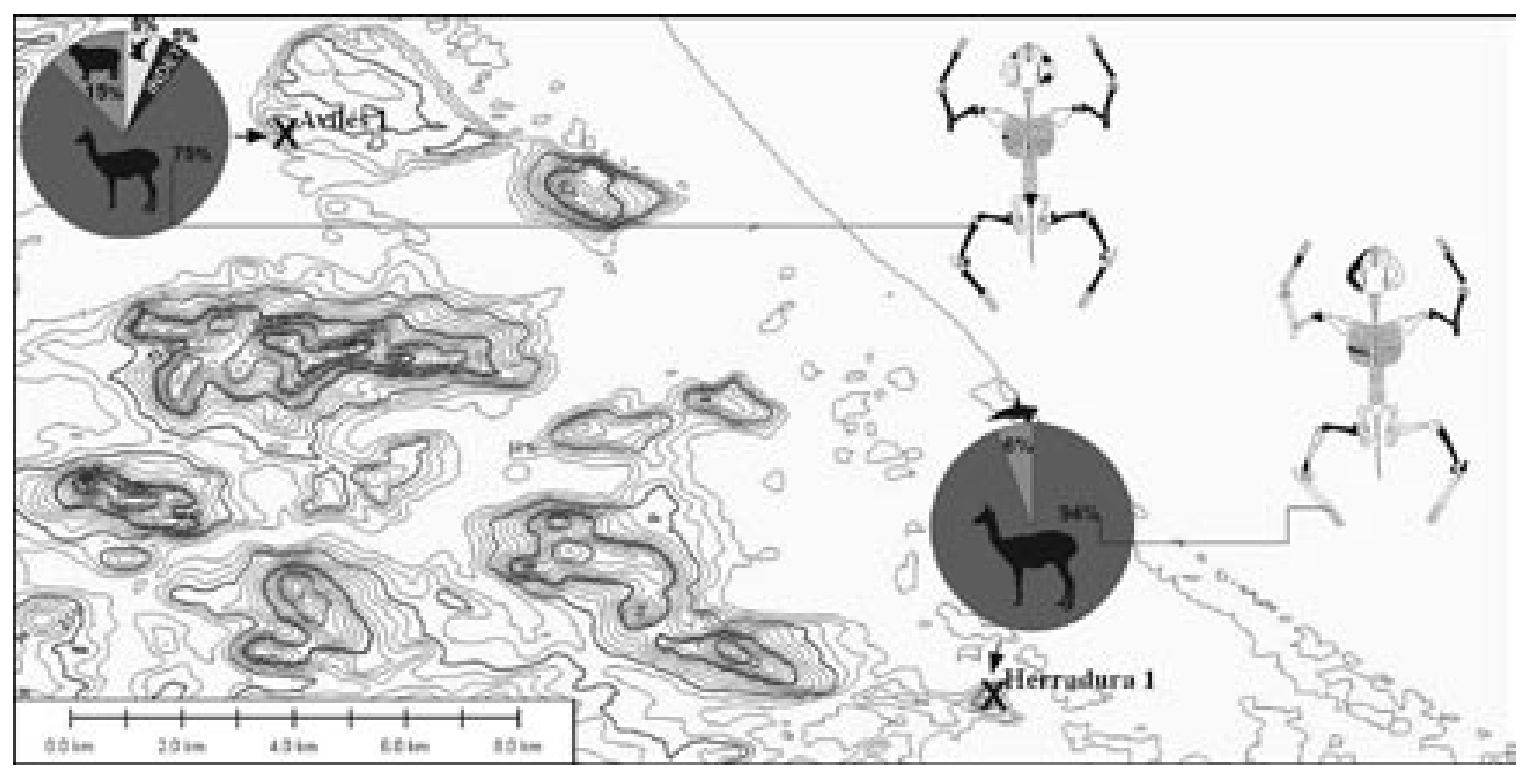

Fig. 4. \%NISP por sitio y representación de partes esqueletales de guanaco.

gicos por la acción del viento, por el escurrimiento producido por el agua de lluvia y de deshielo y por el tránsito de ganado ovino (ver Fig. s/n en Massone et al. 1993: 114). En este proceso los mayores damnificados son los materiales pequeños, tales como microlascas y pequeños fragmentos de huesos.

El sitio A1, desde que se lo localizó hasta el momento en el que se recolectaron los materiales no presentó cambios significativos. Parece encontrarse en un momento de estabilidad. En cambio en el sitio H1 se observaron grandes cambios en su morfología y en la cantidad de materiales expuestos, el proceso de deflación erosionó $980 \mathrm{~m}^{2}$ en aproximadamente 7 meses; el sector norte del sitio, en el cual se encontraba la mayor parte del conjunto óseo, estaba al descubierto. Pero el sector sur, donde se destapó un área de talla lítica, no estaba en superficie en la primera visita del sitio.

Este marcado proceso erosivo es en parte causado por el impacto antrópico presente desde fines del siglo XIX en el área, que ha afectado el paisaje. La introducción del ganado ovino y el alambrado como impedimento a la migración estacional de los animales (nativos), junto al pastoreo continuo de las ovejas, afectó la composición y ecología de las comunidades vegetales nativas en dos sentidos: diversificando algunos tipos de pastizales y degradando otros. De esta forma también fueron afectados los suelos y cuencas de agua (Bianciotto 2006).
En A1 dada la diferencia marcada en los dos perfiles de meteorización en los huesos de guanaco y oveja y la distribución de los restos de oveja dentro del sitio (todos agrupados en un punto), interpretamos que ambas son dos muestras separadas, resultado de dos procesos de acumulación independientes. En los huesos de guanacos podemos observar que los estadios predominantes en orden decreciente son 4, 3 y 5 . Esto implica un mayor tiempo de exposición,

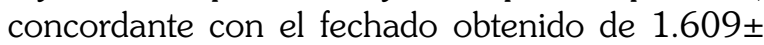
$38^{14} \mathrm{C}$ años AP (AA69653) sobre fémur de guanaco. En los huesos de oveja, además de prevalecer los estadios 1 y 2 , no se evidenciaron fracturas, todos están enteros, los agentes tafonómicos tuvieron menor incidencia y, como ya se dijo, concentrados en un lugar. A considerar por su tamaño, todos los huesos parecen ser de un mismo individuo.

La muestra de guanaco pudo ser producto de la actividad antrópica prehistórica dada la asociación con material lítico y la presencia (escasa) de marcas de origen antrópico. Pero no descartamos que algunos elementos de la muestra hayan ingresado al sitio de manera natural. La muestra de oveja es una depositación reciente, una acumulación de huesos naturales que es parte del ruido de fondo tafonómico regional (Borrero 2000).

Las marcas de raíces en $\mathrm{A} 1$ son el agente tafonómico que ha tenido mayor incidencia en el conjunto de huesos de guanaco (68\% del NISP), 


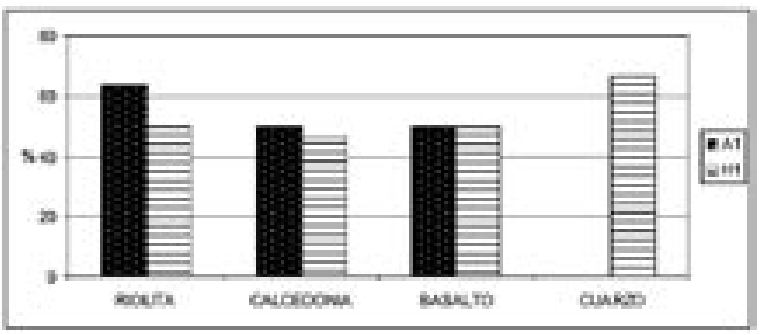

Fig. 5. Índices de corteza de materiales líticos para ambos sitios.

después de la meteorización. Los roedores también han dejado sus marcas en el 32\% de los huesos de guanaco, las mismas se concentran en aristas de diáfisis fragmentadas. Por último, se han hallado marcas ocasionadas por carnívoros, principalmente punctures en el 9\% de la muestra. No se identificaron ahuecados, acanaladuras o piqueteados. Podemos plantear que este actor tafonómico ha tenido poca incidencia sobre la muestra y no ha sido un agente causante de destrucción selectiva de determinados huesos. Es de destacar que la muestra de oveja, solamente tiene marcas de raíces y de roedores, y en menores porcentajes (ver Tabla 4). Esto también estaría avalando que ésta es una incorporación posterior al registro arqueológico.

Con respecto a la representación de especies aprovechadas, se destaca el predominio de guanacos. En H1 la otra especie ingresada (cetáceo) pudo ser introducida como fuente de materia prima ósea, a juzgar por los indicios de trabajo que presenta el especimen. En los restos de guanaco de ambos sitios se puede observar una preponderancia del esqueleto apendicular sobre el axial (Fig. 4).

A los dos conjuntos faunísticos de guanaco se aplicó el test de Spearman para correlacionar las variables \%MAU-\%MGUI y \%MAU-DO (Lyman 1994; Mengoni Goñalons 1999). Se tomaron los valores de DO de guanaco de Elkin (1995) y los de \%MGUI calculados por Borrero (1990), fueron ploteados 32 puntos, teniendo en cuenta las partes presentes en el registro. Ambos conjuntos mostraron correlaciones significativamente positivas entre el \%MAU y el porcentaje del índice general de utilidad modificado (\%MGUI) A1: $\mathrm{R}_{\mathrm{s}}=0,5929$ p >.05; H1: $\mathrm{R}_{\mathrm{s}}=0,5136 \mathrm{p}>.05$ y correlación no significativa entre el \%MAU y la densidad mineral ósea (DO) A1: $\mathrm{R}_{\mathrm{S}}=0,2561 \mathrm{p}>.05 ; \mathrm{H} 1: \mathrm{R}_{\mathrm{S}}=0,3413 \mathrm{p}>$.05. Lo que plantea que la destrucción selectiva mediada por la

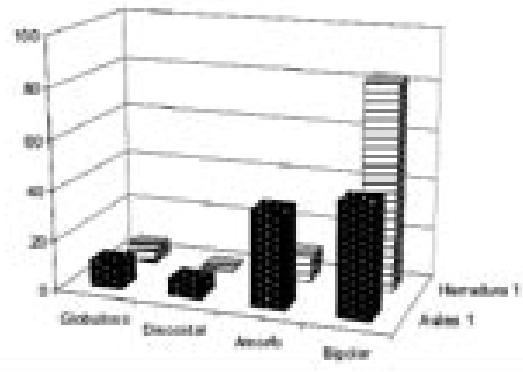

Fig. 6. Tipos de núcleos en porcentajes para ambos sitios.

DO no ha sido la causante de dichas frecuencias, puesto que faltan huesos tanto de alta DO como de baja DO. El factor causal podría ser el transporte selectivo humano, pero también podrían haberse incorporado huesos de guanaco posteriormente, al conjunto óseo.

Los materiales líticos analizados presentan ventajas en relación a otros conjuntos de materiales de superficie, la metodología empleada permitió recuperar todo lo que se hallaba en superficie, sin hacer recolecciones por transectas o muestreos parciales y selectivos, lo cual asegura la representatividad de todos los tipos de restos de los sitios en estudio.

Los instrumentos identificados son principalmente raederas, percutores y lascas con retoque, hay una marcada ausencia de raspadores. La sobre representación de raederas en los conjuntos líticos, o las altas proporciones de las mismas, parece ser una constante en el área de estudio (Santiago, observación personal) y en toda la isla (M. Álvarez com. pers.). Este dato ha sido también observado en muchos sitios arqueológicos de la estepa fueguina del lado chileno de la isla (Massone et al. 1993; Ocampo y Rivas 1996).

La gran cantidad de nódulos, nódulos probados y núcleos nos indica la presencia de fuentes de aprovisionamiento cercanas. El proceso de selección de materia prima, en primer lugar, parece estar orientado por la alta disponibilidad de la misma en el paisaje, y en segundo, por la calidad de la roca para la talla. Otro factor en la selección, puede haber sido la búsqueda de rodados que no requieran muchas operaciones de reducción, para la obtención de la forma base buscada. La preponderancia de riolitas también se ha observado en otros sitios arqueológicos del norte de Tierra del Fuego, tales como La Arcillosa 2 (Salemme et al. 2006), los sitios San Genaro 3 y 4 (Borrazzo 2004), donde son citadas 
como dacitas pero son los mismos tipos, y también en Cabo San Vicente (Morello 2005).

La presencia de corteza es un indicador de las etapas de reducción que se desarrollaron en los sitios. La Fig. 5 muestra los porcentajes de corteza para ambos sitios, discriminados por materias primas. Los mismos son similares a excepción del cuarzo que solamente se presenta en $\mathrm{H} 1$, en forma de nódulos probados.

Como se observa en la Fig. 6 en ambos sitios predominan los núcleos bipolares, y con una mayor preponderancia en H1. Muchos de estos núcleos bipolares solamente fueron testeados y abandonados (lográndose recuperar la totalidad del nódulo original. La mayoría presentaba algún tipo de falla tales como fisuras, poros, cristales de mayor tamaño, impurezas y planos de meteorización y oxidación diferenciales. Es importante remarcar que en $\mathrm{H} 1$ se lograron 9 remontajes dobles, 5 triples y 2 cuádruples, con un total de 42 (9,4\% del total de la muestra) piezas remontadas. En A1 se lograron 8 remontajes dobles, y 1 de forma triple, con un total de 18 piezas (10,8\% del total de la muestra) remontadas. Estos remontajes en su mayoría eran visibles en el campo, aún hace falta más trabajo de laboratorio para agotar las posibilidades de los remontajes físicos. La proporción de remontajes no implica de manera directa que el depósito esté inalterado (Kligmann 1996) sin embargo ninguno de estos remontajes posee fracturas frescas o pátinas diferenciales entre las piezas, así como lascados frescos. Esto puede atribuirse a una baja perturbación de los sitios.

La técnica bipolar o percusión sobre yunque (Morello 2005) casi siempre ha estado asociada a una maximización de un recurso lítico, se la considera como una técnica simple, expeditiva en sí misma; una estrategia adaptada en momentos tales como escasez en el aprovisionamiento de materia prima, el reducido tamaño de las formas base, la falta de tiempo, las condiciones de movilidad (Flegenheimer et al. 1995, Curtoni 1996).

En nuestro caso estaríamos en presencia del proceso contrario: la talla bipolar se utilizó para tallar de forma rápida y expeditiva nódulos de distintos tamaños y calidades. La gran abundancia de materia prima disponible a lo largo y ancho del paisaje, y su regular calidad para la talla, (Santiago et al. 2006) permitió un derroche de la misma. La técnica bipolar se utilizó para conseguir algunas grandes lascas o en su defecto (preferentemente en rodados discoidales) hemiguijarros con extensos filos naturales, para ser transformados en raederas.

Este procedimiento deja una gran cantidad de desechos indiferenciados, y microlascas dando como resultado un escaso aprovechamiento del volumen de los rodados. Las microlascas son las únicas que no están presentes en el registro arqueológico, y esto lo atribuimos a la acción eólica que las habría desplazado de su lugar de depositación original.

Se puede afirmar que en ambos sitios se llevaron a cabo secuencias completas de producción de artefactos líticos con las mencionadas materias primas. Para el sitio $\mathrm{H} 1$ las materias primas cuarzo, microgranito y arenisca se presentan únicamente como débitage. Para el caso de A1, hornblendita y arenisca se encuentran solo como instrumentos (bola y afilador respectivamente). La total ausencia de desechos en estas materias primas nos permite afirmar que estos instrumentos están ingresando al sitio ya manufacturados en otras localidades.

\section{CONCLUSIONES}

En síntesis ambos sitios pueden considerarse como lugares que representan los primeros momentos del procesamiento de carcasas de guanacos y donde se realizaron también actividades relacionada con el testeo de diferentes materias primas líticas. Los conjuntos óseos de ambos sitios presentan algunas evidencias de acción antrópica. Además en estos casos podemos afirmar que la densidad mineral ósea no está mediando en la preservación diferencial de las partes. La conservación de aquellas partes con altos valores de carne, grasa y médula está marcando una clara intencionalidad en la conformación de estos conjuntos. Pero no podemos descartar, la incorporación natural de huesos de guanacos en los sitios, tal como comprobamos la incorporación de huesos de oveja en uno de los mismos.

Ambos casos representan eventos sincrónicos de actividad, esto lo estaría avalando la gran cantidad de piezas líticas que remontan, sin realizarse todavía un análisis de remontajes exhaustivo.

La gran cantidad de desechos indiferenciados, nódulos probados y núcleos bipolares estaría ratificando la práctica de talla bipolar. Esta técnica disminuye el tiempo de selección y abastecimiento 
de materias primas, al permitir aprovechar recursos locales de calidad regular. La estrategia tecnológica utilizada en ambos sitios es expeditiva (sensu Nelson 1991), basada en la anticipación de la presencia de materias primas útiles, y en condiciones previsibles. Las fuentes de aprovisionamiento de las materias primas que fueron utilizadas en $\mathrm{A} 1$ y $\mathrm{H} 1$ son secundarias y presentan una amplia distribución en toda la zona de estudio.

Para el marco cronológico de ambos sitios partiremos del fechado radiocarbónico de $1.609 \pm$ $38^{14} \mathrm{C}$ años AP (AA69653) obtenido de un testigo en A1. El mismo nos está situando en el Holoceno tardío. Por el momento no se tienen fechados absolutos para $\mathrm{H} 1$, pero por encontrarse en la misma posición estratigráfica y por las grandes similitudes en el instrumental con A1, proponemos que también es un sitio del Holoceno tardío.

Estos puntos altos del paisaje parecen ser lugares de uso no recurrentes, por no presentarse palimpsestos de ocupaciones. El tipo de actividades llevadas acabo sobre estos lugares no dejaría grandes acumulaciones de materiales arqueológicos.

La pérdida de información en yacimientos de superficie en el norte de Tierra del Fuego es alta, por la fuerte dinámica de los vientos predominantes, pero consideramos que no por ello debe menospreciarse los datos que contienen este tipo de sitios. Asumimos que la escasa cantidad de microlascas y desechos indiferenciados pequeños se debe a factores postdepositacionales; interpretamos que es principalmente la acción eólica la responsable de la ausencia de este tipo de restos en sitios de superficie. Este agente no puede mover de los lugares de depositación los ítems arqueológicos más grandes y pesados. Es decir, lo que estamos encontrando en estos sitios es solo lo que el viento no se llevó.

\section{AGRADECIMIENTOS}

A la Dra. Mónica Salemme por su constante apoyo, lectura y revisiones críticas del presente trabajo. Al Dr. G. Bujalesky por sus críticas e intercambio de opiniones. Al Dr. D. Acevedo y al Lic. M. González Guillot por la identificación petrológica de los materiales líticos. A la Dra. M. Álvarez por los comentarios en el manuscrito original. A J.P. Pérez y a G. Pinto Vargas por la ayuda en el campo y laboratorio. A J.L. Hormaechea y C. Baldasarre por la ayuda logística en la ciudad de Río Grande. Queremos aclarar, que somos los únicos responsables de lo aquí expresado. Este trabajo ha sido en parte financiado por el PIP-CONICET 6199 concedido a M. Salemme.

\section{BIBLIOGRAFÍA}

ASCHERO, C. 1975 Ensayo para una clasificación morfológica de artefactos líticos aplicada a estudios tipológicos comparativos. Informe presentado al Consejo Nacional de Investigaciones Científicas y Técnicas. Bs. As. 116. Ms.

BEHRENSMEYER, A.K. 1978. Taphonomic and ecologic information from bone weathering. Paleobiology (4): 150-162.

BIANCIOTTO, O. A. 2006. Los Ambientes Naturales de la Estepa Fueguina. Una interpretación de nuestro semidesierto. Dunken. Bs. As.

BORRAZZO, K. 2004. Hacia una tafonomía lítica: el análisis tafonómico y tecnológico de los conjuntos artefactuales líticos de superficie provenientes de los loci San Genaro 3 y 4 (Bahía San Sebastián - Tierra del Fuego, Argentina). Tesis de licenciatura en Ciencias Antropológicas, Facultad de Filosofía y Letras, Universidad de Buenos Aires. Ms.

BORRERO, L. A. 1990. Fuego patagonia bone assemblage and the problem of communal guanaco hunting. En Davies y Reeves (Eds.) Hunter of the recent past. Pp:373-339. Unwin Hyman.

BORRERO, L. A. 2000. Ten years after: esquema para una tafonomía regional de la Patagonia meridional y norte deTierra del Fuego. Desde el País de los Gigantes. Perspectiva Arqueológicas en Patagonia, I. UNPA, Río Gallegos. Pp:183-193.

BORRERO, L. A., J. L. LANATA, B. N. VENTURA. 1992. Distribución de hallazgos aislados en Piedra del Aguila. Análisis espacial en la Arqueología patagónica. Compiladores Borrero, Lanata. Ediciones Ayllu. Pp: 9-20.

BUTZER, K. 1982. Archaeology as Human Ecology. Cambridge University Press, Cambridge.

CURTONI, R.P. 1996. Experimentando con bipolares: indicadores e implicancias arqueológicas. Relaciones de la Sociedad de Antropología. XXI. Bs. As. Pp:187-214.

ELKIN, D. 1995. Volume density of South American camelid skeletal parts. International Journal of Osteoarchaeology. (5):29-37.

FAVIER DUBOIS, C. Y L.A. BORRERO. 2005. Playas de acreción: Cronología y procesos de formación del re- 
gistro arqueológico en la costa central de la bahía San Sebastián, Tierra del Fuego (Argentina). Magallania, (Chile) 33(2):83-98.

FLEGENHEIMER, N., C. BAYÓN, M.I GONZALEZ DE BONAVERI. 1995. Técnica simple, comportamientos complejos: La talla bipolar en la arqueología Bonaerense. Relaciones de la Sociedad de Antropología. XX. Bs. As. Pp: 81-110.

GRAYSON, D. 1984. Quantitative Zooarchaeology. Academic Press. New York.

HOLDAWAY, S.J., SHINER, J. \& FANNING, P. 2004. Huntergatherers and the archaeology of discard behavior: an analysis of surface stone artifacts from Sturt National Park, western New South Wales, Australia. Asian Perspectives 43(1):34-72.

KLIGMANN, D.M., 1996. Ensamblajes líticos y procesos de formación de sitio en Río Pipo 17, un conchero fueguino. En Gómez Otero, J. (ed.); Solo Patagonia: 369-377. Puerto Madryn, Publicación del CEMPAT-CONICET.

LANATA J. L. 1993. Estados alterados: Procesos de formación y conjuntos faunísticos en Rancho Donata, Tierra del Fuego. Explotación de Recursos Faunísticos en Sistemas Adaptativos Americanos. Arqueología Contemporánea (4). Ed. Especial: 163-176.

LYMAN, R. L. 1994. Vertebrate taphonomy. Cambridge Manuals in Archaeology.

MASSONE, M., D. JACKSON, A. PRIETO. 1993. Perspectiva arqueológica de los Selk'nam. Colección de Antropología, Vol. I, Centro de Investigaciones Diego Barros Arana, Dirección de Bibliotecas, Archivos y Museos. Santiago.

MENGONI GOÑALONS, L. 1999 Cazadores de Guanacos de la Estepa Patagónica. Sociedad Argentina de Antropología. Colección Tesis Doctorales. Buenos Aires. Pp: 281.

MORELLO, R, F. 2005. Tecnología y Métodos para el desbaste de lascas en el norte de Tierra del Fuego. Los núcleos del sitio Cabo San Vicente. Magallania, vol.33, no.2, p.29-56. ISSN 0718-2244.
NELSON, M., 1991. The Study of Technological Organization. En Schiffer, M. (ed.) Archaeological Method and Theory 3:57-100. Tucson, The University of Arizona Press.

OCAMPO, C.E. Y P.H. RIVAS, 1996. Caracterización arqueológica preliminar del suroeste de Tierra del Fuego. ANS. INST. PAT. Ser. Cs. Hs. Punta Arenas. (24):125-151.

OLIVA, G., L. GONZÁLEZ, P. RIAL, E. LIVRAGHI. 2001. Áreas Ecológicas de Santa Cruz y Tierra del Fuego.

Ganadería Ovina Sustentable en la Patagonia Austral. INTA. Río Gallegos.

ORQUERA, L. Y E. PIANA. 1986. Normas para la descripción de objetos arqueológicos de piedra tallada. CADIC, Contribución Científica (Publicación especial) $\mathrm{N}^{\circ} 1$, Ushuaia. Pp: 198

PRIETO, A. 1997. Algunos resultados de los trabajos arqueológicos en Juni Aike 2. Anales del Instituto de la Patagonia. Serie Cs. Hs., Punta Arenas (Chile). (25):137-146.

RAMOS, M. Y J. MERENZON. 2004. Ensamblajes, tecnología y análisis espacial del primer componente de Túnel I. Temas de Arqueología, Análisis lítico. A. Acosta, D. Loponte y M. Ramos Comp. Pp:145-191.

SALEMME, M., G. BUJALESKY. Y F. SANTIAGO, 2006. La Arcillosa 2: la ocupación humana durante el Holoceno medio en el Río Chico, Tierra del Fuego, Argentina. Sextas Jornadas de Arqueología de la Patagonia. Punta Arenas. Trabajo completo aceptado para publicación.

SANTIAGO F. C., G. G. BUJALESKY Y M. C. SALEMME. 2006. Prospección Arqueológica en la cuenca del Río Chico. Tierra del Fuego. Argentina. Sextas Jornadas de Arqueología de la Patagonia. Punta Arenas. Pp: 1-18. Trabajo completo aceptado para publicación.

ZÁRATE, M.A., M.I. GONZÁLEZ DE BONAVERI, N. FLEGENHEIMER, C. BAYÓN. 2000-2002. Sitios arqueológicos someros: El concepto de sitio en estratigrafía y sitio de superficie. Cuadernos del Instituto de Antropología y Pensamiento Latinoamericano. (19) ISSN 0570-8346. Pp: 635-653. 